\title{
The Automatically Analyzed (AA) ColonView (CV) Quick Test for Fecal Occult Blood Shows Higher Diagnostic Accuracy in Detection of Colorectal Adenoma than Visually Analyzed Tests
}

\author{
JANNICA MEKLIN ${ }^{1 *}$, MAARET ESKELINEN ${ }^{1 *}$, DENISE PEIXOTO GUIMARAES ${ }^{2}$, \\ TUOMAS SELANDER ${ }^{3}$, TAPANI TIUSANEN ${ }^{4}$, KARI SYRJÄNEN $^{2,5}$ and MATTI ESKELINEN ${ }^{1}$ \\ ${ }^{1}$ Department of Surgery, Kuopio University Hospital and School of Medicine, \\ University of Eastern Finland, Kuopio, Finland; \\ ${ }^{2}$ Molecular Oncology Research Center, Barretos Cancer Hospital, Barretos, Brazil, \\ ${ }^{3}$ Science Service Center, Kuopio University Hospital and School of Medicine, \\ University of Eastern Finland, Kuopio, Finland; \\ ${ }^{4}$ Department of Clinical Research, Biohit Oyj, Helsinki, Finland; \\ ${ }^{5}$ SMW Consultants, Ltd., Kaarina, Finland
}

\begin{abstract}
Background/Aim: The present study compared the accuracy of visually analyzed (VA) and automatically analyzed (AA) ColonView (CV) quick test; a new-generation fecal immunochemical test (FIT) for hemoglobin ( $\mathrm{Hb}$ ) and hemoglobin/haptoglobin (Hb/Hp) (Biohit Oyj, Helsinki, Finland) in subjects participating in colorectal neoplasia (CRN) detection in Brazil. A traditional guaiac-based fecal occult blood test (gFOBT) test (HemoccultSENSA) was used as a reference. Patients and Methods: A cohort of 509 colonoscopyreferral patients were asked to collect three consecutive fecal samples, to be analyzed by both CV and SENSA. Results: In ROC analysis for the AA reading, the optimal cut-off value for $C V$ Hb was $\geq 8.0912$ and that for $C V H b / H p$ was $\geq 1.8983$. With these cut-offs, the sensitivity (Se), specificity (Sp), and efficiency of $C V A A$ in detecting colorectal adenoma (CRA) were: $64.2 \% / 78.6 \%, 53.4 \% / 35.3 \%$, and $58.6 \% / 56.5 \%$, for $\mathrm{Hb}$ and $\mathrm{Hb} / \mathrm{Hp}$, respectively. In the HSROC analysis, the AUC values for i) VA and ii) AA modes were as follows: i) $A U C=0.551$ (95\%CI $=0.500-0.602)$, ii) $A U C=0.606$ (95\%CI $=0.550-0.662$ ).
\end{abstract}

This article is freely accessible online.

*These Authors contributed equally to this study.

Correspondence to: Matti Eskelinen, MD, Ph.D., School of Medicine, University of Eastern Finland, P.O. Box 100, FI-70029 KYS, Finland. Tel: +358 17173311, Fax: +358 17172611, GSM: +358 400969444, e-mail: matti.eskelinen@kuh.fi

Key Words: Fecal occult blood, fecal immunochemical test, FIT, colorectal adenoma screening, sensitivity, specificity, false negative, false positive, ROC, HSROC.
The difference between these AUC values was statistically significant ( $p=0.0160)$. Conclusion: The present study confirms the previous results on the applicability of the ColonView quick test in CRN screening. Of the two optional reading modes, the $A A$ reading showed significantly better diagnostic accuracy as compared to the VA reading (or SENSA), in detecting the CRA endpoint in colonoscopy-referral patients.

Although, several randomized trials have shown a decrease in colorectal cancer (CRC)-related mortality in patients who undergo screening with guaiac-based fecal occult blood testing (gFOBT) (1-6), the clinical trials have demonstrated that the diagnostic performance of FITs is superior to standard gFOBTs in detecting colorectal neoplasia (CRN) (7-12). Recent studies have also confirmed that using the FITs results in higher participation rates in CRC screening, because of a simplified sampling protocol, not interfered by the dietary restrictions necessary for the gFOBTs $(7,9,11-13)$. Other important issues to be considered while selecting the test for CRC screening include i) the ideal cut-off value for hemoglobin $(\mathrm{Hb})$ detection, ii) the relative value of qualitative and quantitative tests, as well as iii) the number of samples needed to reach an optimal sensitivity and specificity. According to the European Guidelines for quality assurance in CRN screening (7), another advantage of the FITs in population screening is that these tests can be automated and the end user can adjust the cut-off at which a positive result is reported (14). Although, an increasing number of FITs have been developed and are available on the market, relatively few of those have been tested for diagnostic accuracy in CRC screening. Current data imply, however, that new FITs have superior test characteristics as compared with gFOBTs $(7,15,16)$. 


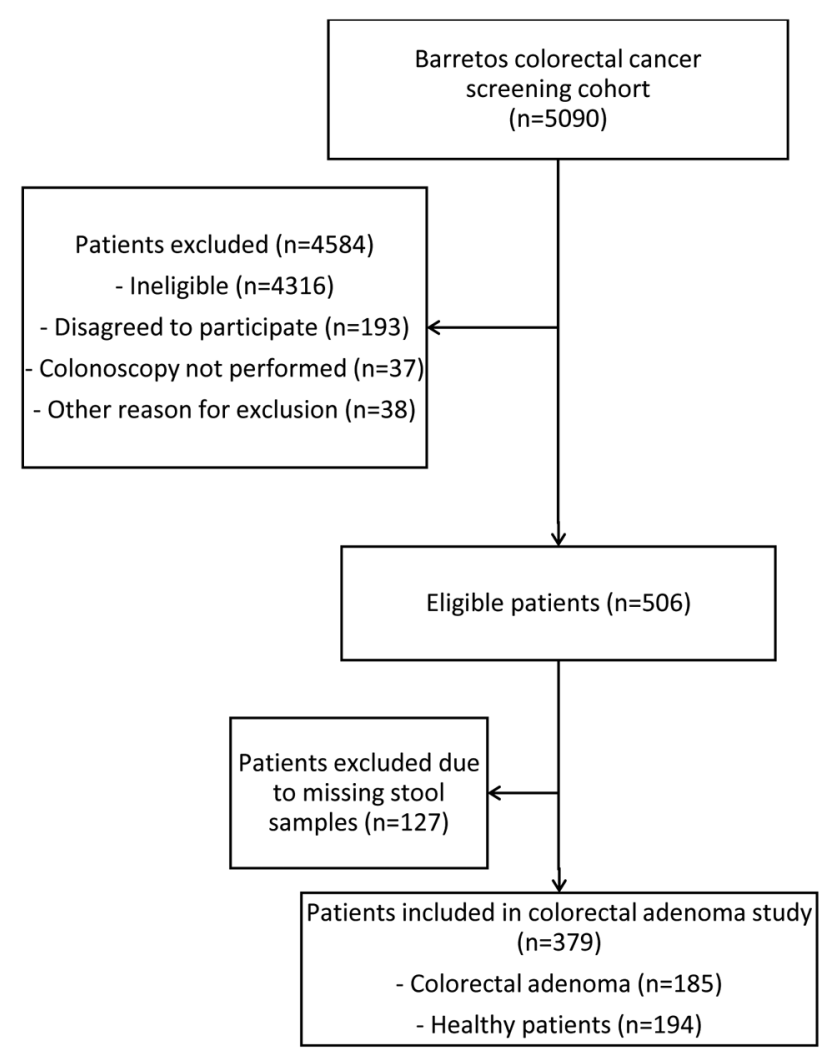

Figure 1. Flow-chart of the study.

Vasilyev et al. (11) and Guimaraes et al. (12) have previously compared the diagnostic accuracy of HemoccultSENSA (SENSA) and CV in a head-to-head comparison study among CRN screening patients. The present study is an extension of these analyses by applying hierarchical summary receiver operating characteristic (HSROC) and area under curve (AUC) analyses to test different cut-offs and to compare the diagnostic accuracy of the two reading modes of CV: Visual analysis (VA) and automatic analysis (AA), using colorectal adenoma (CRA) as the endpoint in patients referred to colonoscopy for detection of CRN.

\section{Patients and Methods}

The Barretos CRN screening cohort included 5,090 subjects, but 4,316 patients were ineligible for the study, 193 patients disagreed to participate, in 37 patients colonoscopy was missing and 38 patients had other reason for exclusion (Figure 1). Three fecal samples were requested and all subjects underwent diagnostic colonoscopy with biopsy confirmation. The study protocol and inclusion/exclusion criteria of study patients were detailed in a previous report by Guimaraes et al. (12).

Sample collection, processing and interpretation of results. A newgeneration FIT, ColonView ${ }^{\circledR}$ quick test (subsequently CV) (Biohit Oyj) does not necessitate any preparatory steps of the patient or compliance with any restrictions in the daily diet or medication. The guaiac-based traditional FOBT (Hemoccult SENSA, Beckman Coulter Inc., Passadena, CA, USA) was used as the reference test in this study. The sample collection protocol of both tests was described in more detail recently (12).

For the $\mathrm{CV}$, two optional reading modes are available: visual analysis (VA) and automatic analysis (AA). The latter is performed by using opTrilyzer Lateral flow reader (Chembio Diagnostics $\mathrm{GmbH}$, Berlin, Germany), as described before $(11,12)$. In fully compliant patients, three stool samples were tested by $\mathrm{CV}$ and the result was interpreted positive if any of the three samples tested positive for either $\mathrm{Hb}$ or $\mathrm{Hb} / \mathrm{Hp}$ complex. The analytical sensitivity for $\mathrm{CV} \mathrm{Hb}$ is $15 \mathrm{ng} / \mathrm{ml}$, and for $\mathrm{CV} \mathrm{Hb} / \mathrm{Hp}$ complex, $4 \mathrm{ng} / \mathrm{ml}$ (17).

Normal colonoscopy was used as the gold standard indicating a negative result regarding the study endpoints, as described before (12).

Statistical analysis. STATA/SE version 17.0 (StataCorp, College Station, TX, USA) was used for analysis. The statistical tests presented were two-sided, and $p$-values $<0.05$ was considered statistically significant. Using $2 \times 2$ tables, sensitivity (Se) and specificity ( $\mathrm{Sp}$ ) with $95 \%$ confidence intervals $(95 \% \mathrm{CI})$ for each test was determined. Conventional ROC analysis was used to graph for $\mathrm{Se}$ and $\mathrm{Sp}$ as well as to find the optimal cut-off values for both $\mathrm{Hb}$ and $\mathrm{Hb} / \mathrm{Hp}$ of the $\mathrm{CV}$ test. Meta-analytical technique (metaprop) was used to create separate forest plots for Se and Sp, with each set of data included (i.e., test components $\mathrm{Hb}, \mathrm{Hb} / \mathrm{Hp}$, cut-offs). We also calculated the summary estimates of Se and Sp, positive (LR+) and negative likelihood ratio (LR-) as well as diagnostic odds ratio, using a random effects bivariate model and fitted the summary hierarchical receiving operating characteristic (HSROC) curves for the CRA as the endpoint. Roccomp test was used to compare the statistical significance between the AUC (area under the curve) values of the AA and VA reading modes.

\section{Results}

Patient data of the study. The whole screening cohort at Barretos Cancer Hospital (BCH) included 5,090 patients, but due to various reasons, 4,584 of the subjects had to be excluded (Figure 1). There were 506 eligible patients for the study, of whom 127 patients were excluded due to missing stool samples. The final cohort included 379 colonoscopyreferral patients evaluated by the $\mathrm{VA}$ and $\mathrm{AA}$ reading modes of the CV tests and SENSA.

VA screening tests for colorectal adenoma endpoint. The Se, $\mathrm{Sp}$ and efficiency of the SENSA test detecting CRA were as follows: $21.1 \%, 83.0 \%, 52.8 \%$ (Table I). The Se, Sp and efficiency of the visually analyzed $\mathrm{CV} \mathrm{Hb}$ and $\mathrm{CV} \mathrm{Hb} / \mathrm{Hp}$ tests detecting CRA were as follows: 46.9\%/43.6\%, 64.0\%/60.6\%, $55.7 \% / 52.3 \%$ (Table I). The PV+ of CV Hb VA test (Table I) was slightly higher than that of test $\mathrm{CV} \mathrm{Hb/Hp} \mathrm{VA} \mathrm{test} \mathrm{(Table}$ II); $55.0 \%$ versus $51.6 \%$, indicating the proportion of the patients with a positive test result who have the disease. When $\mathrm{SENSA}+\mathrm{CV} \mathrm{Hb}+\mathrm{Hb} / \mathrm{Hp}$ VA (Table I) were used as a combined test panel for the CRA endpoint, the panel had $52.7 \%$ Se, $54.3 \% \mathrm{Sp}$ and $53.5 \%$ efficiency (Table I). 
Table I. Visually analyzed screening tests for the colorectal adenoma endpoint.

\begin{tabular}{|c|c|c|c|c|c|c|c|}
\hline Test number & $\begin{array}{l}\text { Fecal occult } \\
\text { blood tests }\end{array}$ & $\begin{array}{l}\text { Positive endpoint } \\
\text { (colorectal adenoma) }\end{array}$ & $\begin{array}{c}\text { Negative endpoint } \\
\text { (no colorectal adenoma) }\end{array}$ & $\mathrm{TP}$ & FN & FP & $\mathrm{TN}$ \\
\hline VA 1 & HemoccultSENSA & Test positive & Test negative & 39 & 146 & 33 & 161 \\
\hline VA 2 & ColonView Hb VA & Test positive & Test negative & 83 & 94 & 68 & 121 \\
\hline VA 3 & ColonView $\mathrm{Hb} / \mathrm{Hp}$ VA & Test positive & Test negative & 79 & 102 & 74 & 114 \\
\hline VA 4 & ColonView $\mathrm{Hb}+\mathrm{Hb} / \mathrm{Hp}$ VA & One or more sample positive & All samples negative & 92 & 89 & 79 & 110 \\
\hline VA 5 & ColonView $\mathrm{Hb}+\mathrm{Hb} / \mathrm{Hp}$ VA+SENSA & One or more sample positive & All samples negative & 99 & 89 & 90 & 107 \\
\hline
\end{tabular}

FN: False-negative; FP: false-positive; TN: true negative; TP; true positive; VA: visually analyzed.

Table II. Automatically analyzed screening tests for the colorectal adenoma endpoint.

\begin{tabular}{|c|c|c|c|c|c|c|c|}
\hline Test number & $\begin{array}{l}\text { Fecal occult } \\
\text { blood tests }\end{array}$ & $\begin{array}{l}\text { Positive endpoint } \\
\text { (colorectal adenoma) }\end{array}$ & $\begin{array}{c}\text { Negative endpoint } \\
\text { (no colorectal adenoma) }\end{array}$ & $\mathrm{TP}$ & FN & FP & $\mathrm{TN}$ \\
\hline AA 1 & ColonView Hb AA & $\geq 9.8796$ (median) & $<9.8796$ & 103 & 76 & 82 & 109 \\
\hline AA 2 & ColonView $\mathrm{Hb} / \mathrm{Hp} \mathrm{AA}$ & $\geq 8.1592($ median $)$ & $<8.1592$ & 102 & 80 & 84 & 106 \\
\hline AA 3 & ColonView Hb AA & $\geq 8.0912(\mathrm{ROC})$ & $<8.0912(\mathrm{ROC})$ & 115 & 64 & 89 & 102 \\
\hline AA 4 & ColonView $\mathrm{Hb} / \mathrm{Hp} \mathrm{AA}$ & $\geq 1.8983(\mathrm{ROC})$ & $<1.8983$ (ROC) & 143 & 39 & 123 & 67 \\
\hline AA 5 & ColonView $\mathrm{Hb}+\mathrm{Hb} / \mathrm{Hp} \mathrm{AA}$ & Test positive* & Test negative & 152 & 30 & 133 & 59 \\
\hline
\end{tabular}

FN: False-negative; FP: false-positive; TN: true negative; TP: true positive; VA: visually analyzed; AA: automatically analyzed. *Cut-offs for positive tests for CV Hb AA $\geq 8.0912$ and $\mathrm{CV} \mathrm{Hb/Hp} \mathrm{AA} \geq 1.8983$.

ROC analysis and optimal cut-off values of the $C V$. The ROC analysis showed the optimal cut-off value of $\geq 8.0912$ for $\mathrm{CV} \mathrm{Hb}$ AA (Table II) and $\geq 1.8983$ for $\mathrm{CV} \mathrm{Hb} / \mathrm{Hp} \mathrm{AA}$ (Table II). Using these cut-offs in the AA mode, the Se, Sp and efficiency of the $\mathrm{Hb}$ (Table II) and $\mathrm{Hb} / \mathrm{Hp}$ (Table II) tests detecting CRA were as follows: 64.2\%/78.6\%, $53.4 \% / 35.3 \%$ and $58.6 \% / 56.5 \%$. The PV+ of CV Hb AA test (Table II) was slightly higher than that of test CV $\mathrm{Hb} / \mathrm{Hp}$ AA test (Table II); $56.4 \%$ versus $53.8 \%$, indicating the proportion of the patients with a positive test result who have the disease. When $\mathrm{CV} \mathrm{Hb}+\mathrm{Hb} / \mathrm{Hp} \mathrm{AA}$ (Table II) was used as a combined test panel for the CRA endpoint, the panel had $83.5 \%$ Se, $30.7 \%$ Sp and $56.4 \%$ efficiency (Table II). Figure 2 shows the AUC values in ROC analysis of the $\mathrm{CV} \mathrm{Hb} \mathrm{AA} \mathrm{and} \mathrm{CV} \mathrm{Hb/Hp} \mathrm{AA} \mathrm{tests} \mathrm{for} \mathrm{the} \mathrm{CRA} \mathrm{endpoint:}$ 0.605 and 0.590 , respectively.

Diagnostic accuracy of the $C V$ test in the VA mode. The overall Se of the CV in the VA mode for detecting CRA was $43 \%(95 \% \mathrm{CI}=31-55 \%)$ (Figure 3$)$. The three most sensitive $\mathrm{CV}$ test VA panels (CV Hb VA, CV $\mathrm{Hb}+\mathrm{Hb} / \mathrm{Hp} \mathrm{VA}$, and $\mathrm{CV}+$ SENSA panel) showed 47-53\% Se (Figure 3). The overall $\mathrm{Sp}$ of the VA mode was $64 \%(95 \% \mathrm{CI}=54-75 \%)$ (Figure 4). The three most specific VA test modes showed Sp values of $61-83 \%$ (Figure 4 ).

Diagnostic accuracy of the $C V$ test in the AA mode. The overall $\mathrm{Se}$ of the AA reading mode for detecting CRA was
$69 \%(95 \% \mathrm{CI}=57-79 \%)$ (Figure 5). The two most sensitive AA tests (CV Hb/Hp AA at cut-off $\geq 1.8983$, and $\mathrm{CV} \mathrm{Hb}+$ $\mathrm{Hb} / \mathrm{Hp}$ AA test panel) showed $79-84 \%$ Se (Figure 5). The overall $\mathrm{Sp}$ of the AA reading mode for the CRA endpoint was $46 \%(95 \% \mathrm{CI}=35-57 \%)$ (Figure 6). The three most specific AA tests (CV Hb AA at cut-off $\geq 9.8796, \mathrm{CV} \mathrm{Hb/Hp} \mathrm{AA} \mathrm{at}$ cut-off $\geq 8.1592$ and $\mathrm{CV} \mathrm{Hb}+\mathrm{Hb}$ AA at cut-off $\geq 8.0912$ ) in CRA diagnosis showed an Sp range of 53-57\% (Figure 6).

HSROC and AUC values. HSROC curves were used to visualize the pooled overall accuracy of VA (Figure 7) and AA (Figure 8) reading modes in CRA detection. In HSROC analysis, the AUC values for i) VA, and ii) AA modes were as follows: i) $\mathrm{AUC}=0.551(95 \% \mathrm{CI}=0.500-0.602)$ (Figure 7), and ii) $\mathrm{AUC}=0.606(95 \% \mathrm{CI}=0.550-0.662)$ (Figure 8). The difference between these AUC values (Roccomp analysis) was statistically significant $(p=0.0160)$.

\section{Discussion}

FITs have been developed to solve the analytical problems associated with the traditional gFOBTs. Our aim was to test the performance of visually analyzed (VA) and automatically analyzed (AA) new-generation FIT for $\mathrm{Hb}$ and $\mathrm{Hb} / \mathrm{Hp}$ (ColonView quick test) in subjects participating in $\mathrm{CRN}$ detection in Brazil. A traditional gFOBT test (SENSA) was used as the reference test. 


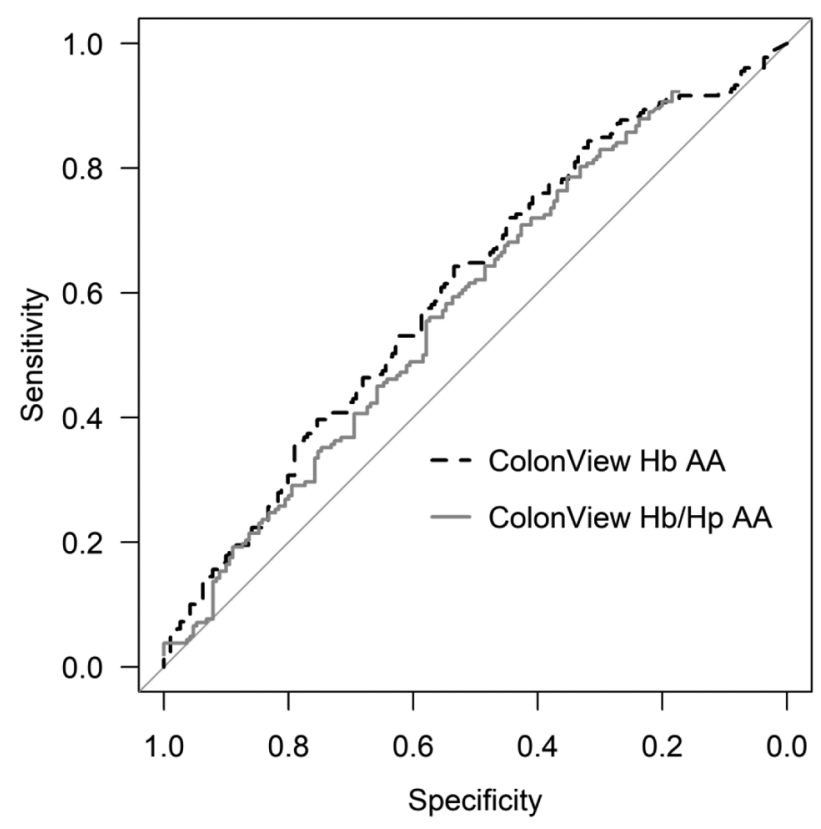

Figure 2. Receiver operating characteristic (ROC) curve for test optimization and finding the optimal cut-off point for the automatically analyzed (AA) ColonView (CV) test for the colorectal adenoma endpoint.

For reading of the $\mathrm{CV}$ test results, two optional modes are available: VA and AA (18). For the automatic reading, the Quick Test Reader (QTR) is needed. QTR is a mobile device for quantitative evaluation of lateral flow assays. The device is initially tested and configured by the original device manufacturer (opTricon $\mathrm{GmbH}$ ), and subsequently validated and configured by Biohit (Biohit Oyj) for the use in CV AA reading (18). In this calibration, VA and AA are interlinked in that the weakest band visualised as positive (by several observers) was equivalent to the reader AA value of $20 \mathrm{ng} / \mathrm{ml}$ (18).

In quantitative tests, the optimal cut-off levels for $\mathrm{Hb}$ detection depend on the desired tradeoff between sensitivity and false-positive (FP) rate. This can be neatly done by using conventional non-parametric ROC analysis $(19,20)$, selecting the coordinate points in the ROC curve as indicators of these cut-off values, separately for $\mathrm{Hb}$ and the $\mathrm{Hb} / \mathrm{Hp}$ complex. The cut-off values have become increasingly important with the introduction of quantitative iFOBTs in which it is possible to adjust the cut-off limit to obtain an acceptable compromise between clinical sensitivity and specificity.

The ideal balance between Se and Sp in FOBT depends on the screening cohort, and FP rate is associated with an increased number of unnecessary colonoscopies (21). Therefore, an optimal FOBT for CRN screening will provide an opportunity for quantitative optimization of the cut-off values. While the choice of FOBT for CRN screening mainly focuses on diagnostic accuracy, other associated factors also require consideration including test costs and compliance. A recent study on longitudinal adherence of the patients with CRN screening in a primary care cohort showed that less than 50 percent of the patients completed gFOBT (SENSA) screening during the 2-year follow-up period (22). Therefore, a significant advantage of the FITs is an improved screening compliance as compared to gFOBTs. In addition, the use of gFOBTs include complicated dietary restrictions, leading to poor test compliance among the test subjects $(15,16)$. As to the CV test, and particularly its AA reading mode, the test offers a number of other advantages in CRN screening, such as integration into the laboratory information system and the opportunity for quality control $(11,12,15,16)$.

The present study is limited by its design that does not enable us to predict whether enhanced Se of the CV test for detection of CRA will result in a decreased mortality rate in CRC. While our study sample has an effect on the pretest probability of CRA, our CRA detection rate of eligible patients in CRC screening cohort 185/506 (36.6\%) was slightly higher than that of earlier CRA screening cohorts (23-25).

The FIT tests were recently subjected to a meta-analysis by Lee et al. (26) and Meklin et al. (16). Interestingly, in these meta-analyses the authors used only the CRC endpoint while pooling the FIT results. This decision was based on their observation that only a subset of all studies reported adequate data on CRA and that there was a wide variability in the definitions of adenomas $(16,26)$. Thus, all studies of CRA were excluded from these two meta-analyses. However, the conclusion was that the $\mathrm{CV}$ quick test is superior to the traditional gFOBTs in screening $(15,16)$.

There are three previous screening cohorts available where the CRA endpoint was used (23-25). Allison et al. (23) assessed 139 patients with advanced neoplasms (14 CRC, 128 CRA) and found $29.5 \%$ Se for FIT and $22.8 \%$ Se for panel of SENSA and FIT. However, they did not use HSROC and AUC analysis (27-31) to confirm the diagnostic performance of the tests in these patients. Hundt et al. (24) collected stool samples before colonoscopy of the patients with CRA and tested the diagnostic performance of six different FITs. They reported a pooled Se of 25\% $(95 \%$ $\mathrm{CI}=18-34 \%$ ) for the CRA endpoint. Similar to Allison et al. (23), the authors did not measure the diagnostic accuracy of the FITs using the HSROC analysis (24). In addition, this study deviated from the real-life conditions in that the stool samples were not directly stored in a buffer vial, but frozen before testing instead.

The only previous study where both $\mathrm{Hb}$ and $\mathrm{Hb} / \mathrm{Hp}$ complex are included is the report of Haug et al. (25), who used automated ELISA (RIDASCREEN) to study a cohort of 130 patients with advanced adenoma. Being one of few tests 


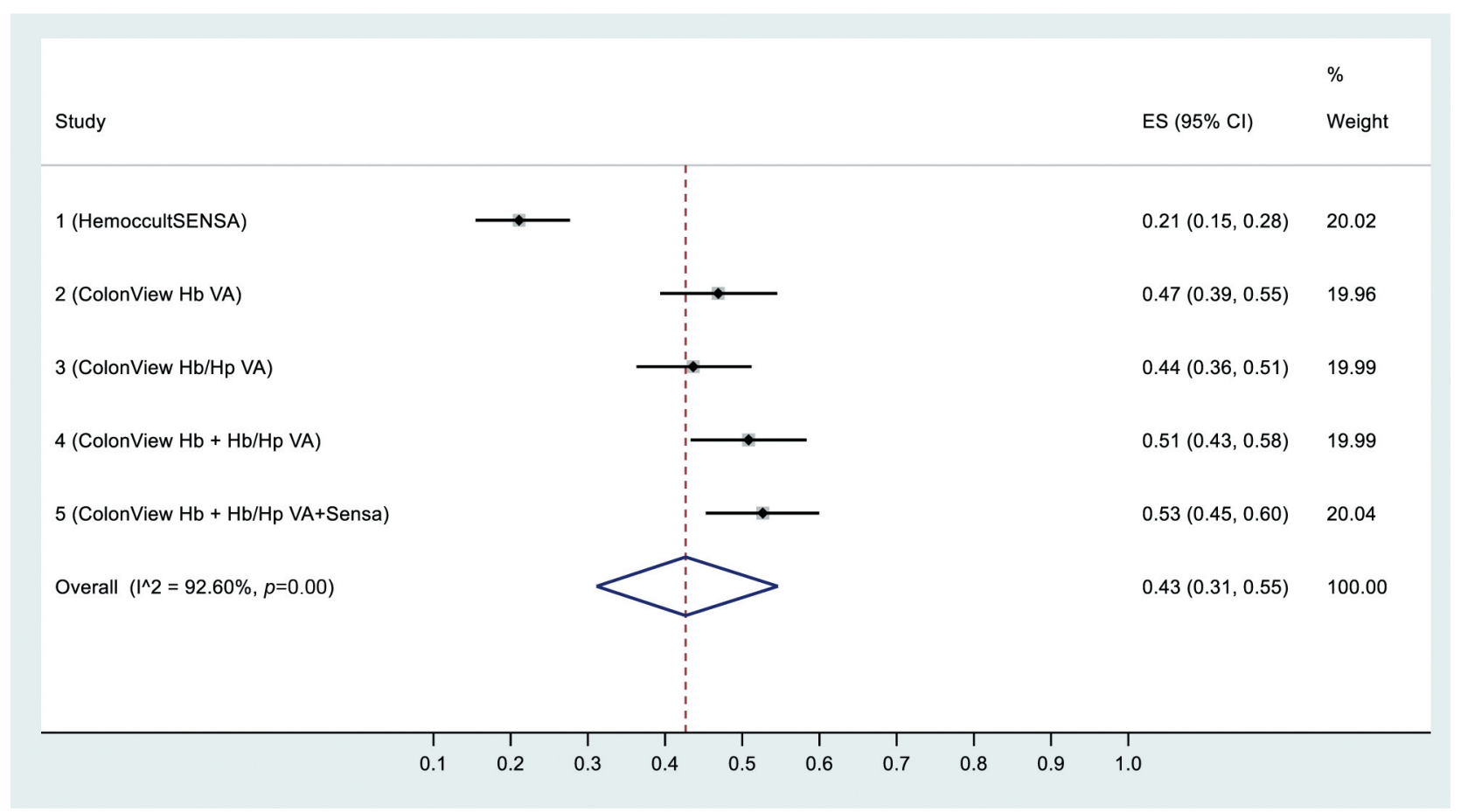

Figure 3. Sensitivity values of visually analyzed (VA) screening tests for the colorectal adenoma endpoint. ES: Estimated sensitivity; CI: confidence interval.

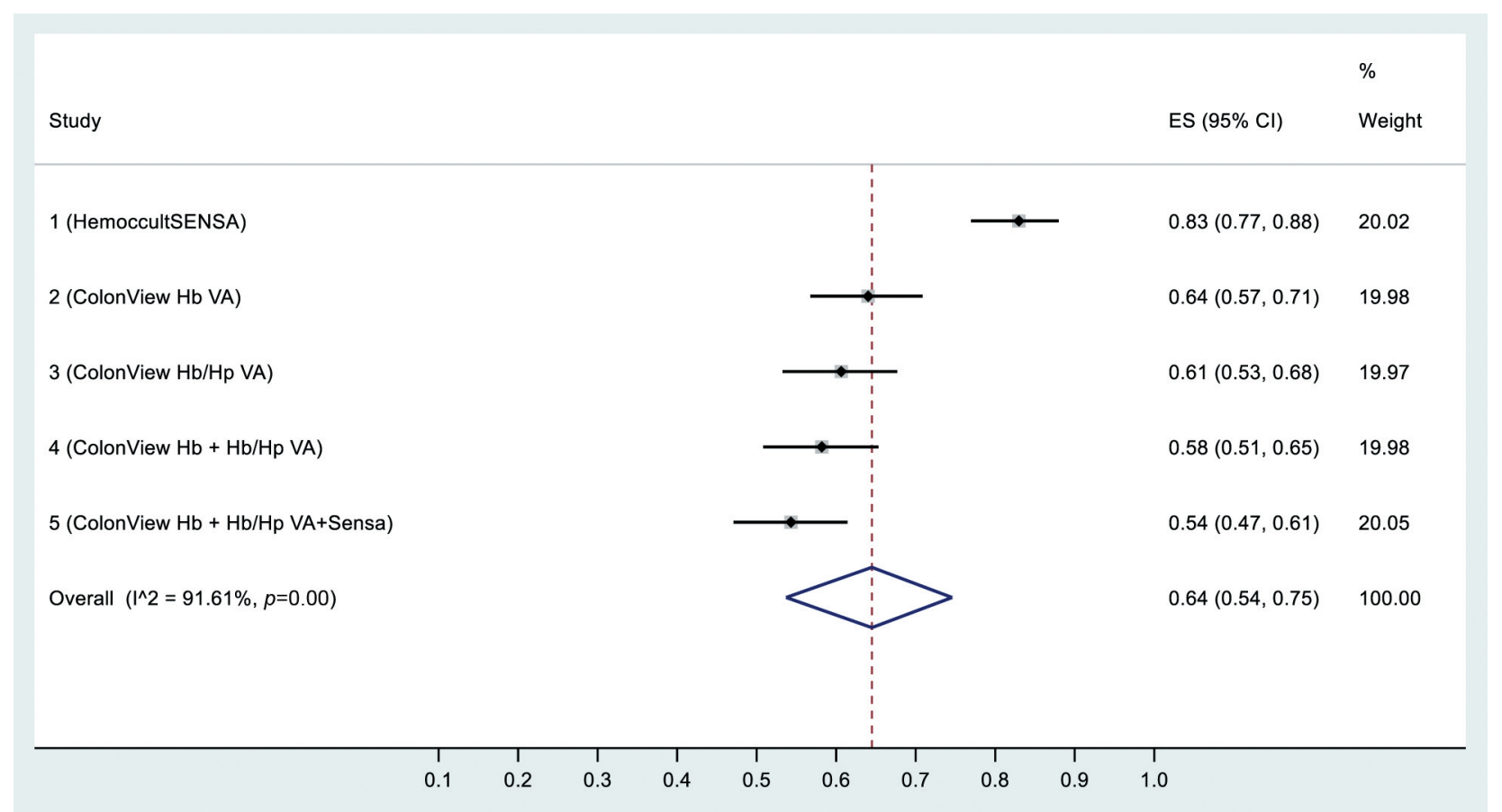

Figure 4. Specificity values of visually analyzed (VA) screening tests for the colorectal adenoma endpoint. ES: Estimated specificity; CI: confidence interval. 


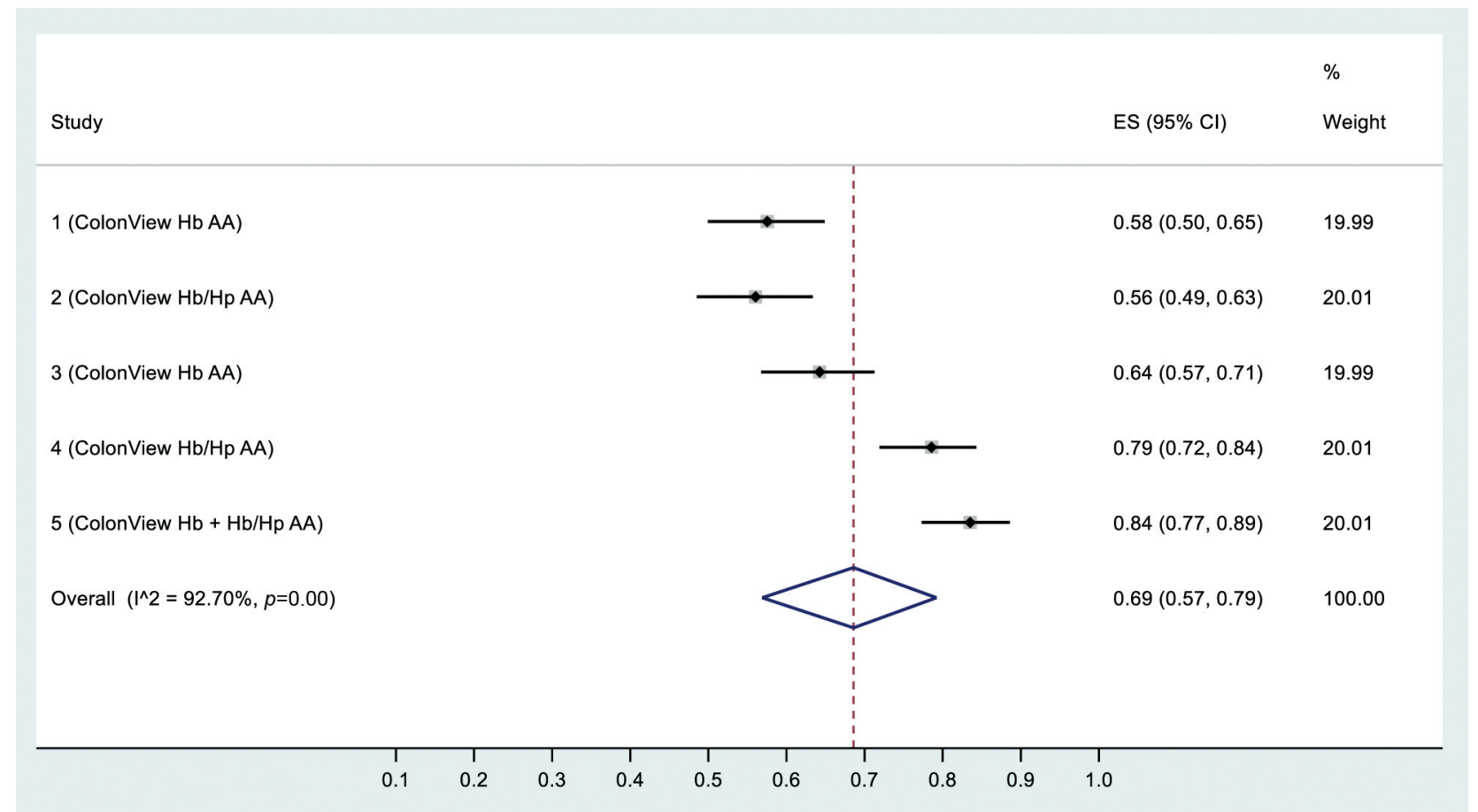

Figure 5. Sensitivity values of automatically analyzed (AA) screening tests for the colorectal adenoma endpoint. ES: Estimated sensitivity; CI: confidence interval.

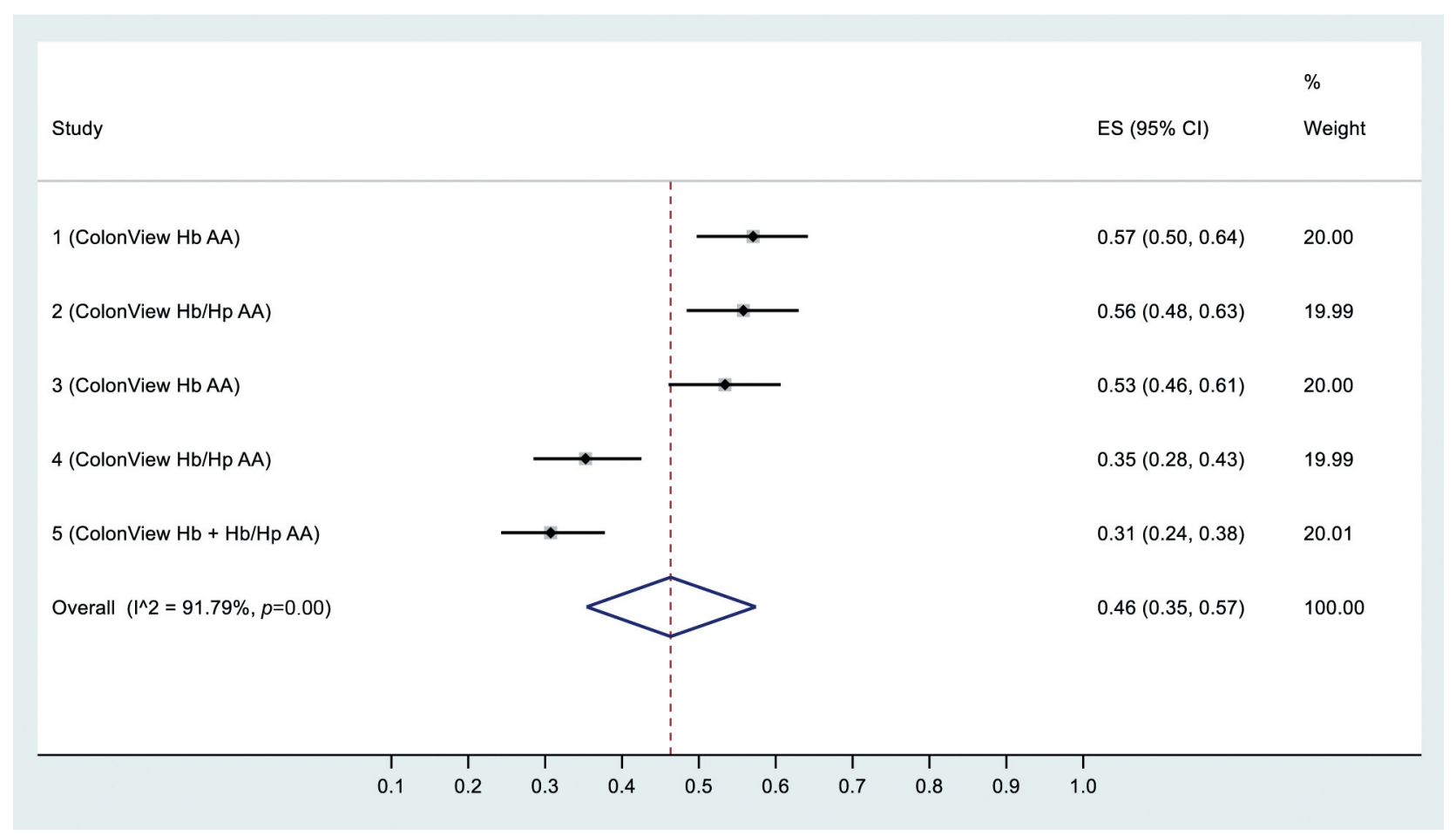

Figure 6. Specificity values of automatically analyzed (AA) screening tests for the colorectal adenoma endpoint. ES: Estimated specificity; CI: confidence interval. 


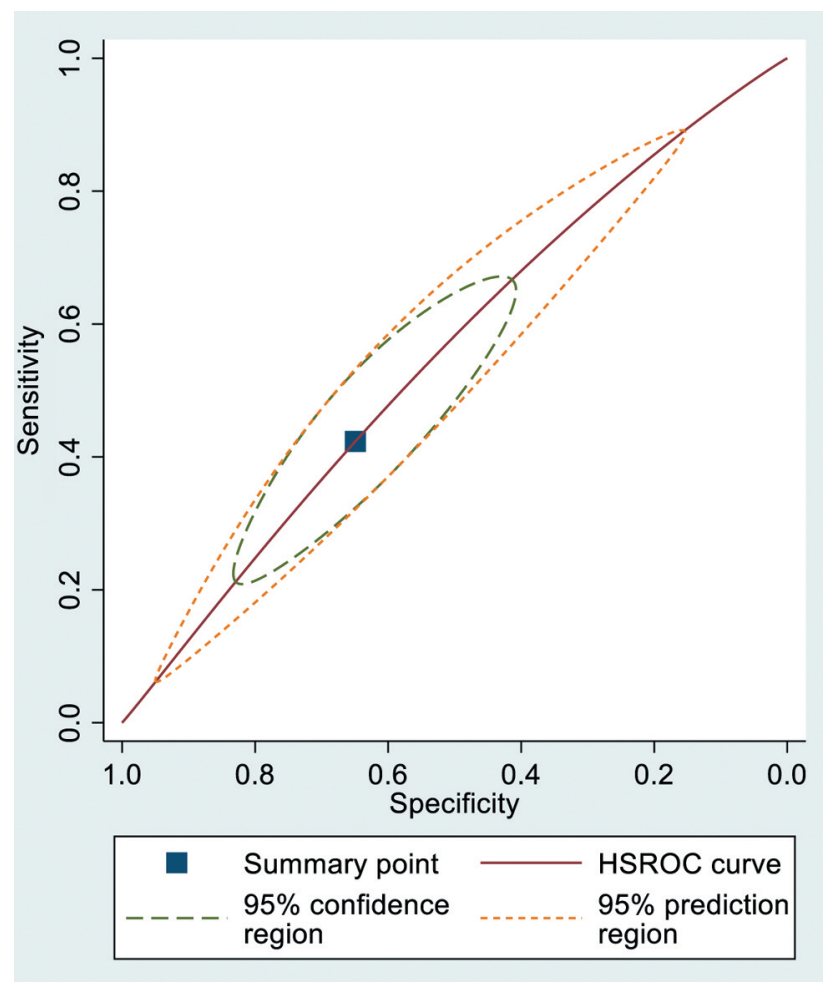

Figure 7. Hierarchical summary receiver operating characteristic (HSROC) curve of the visually analyzed (VA) screening tests for the colorectal adenoma endpoint.

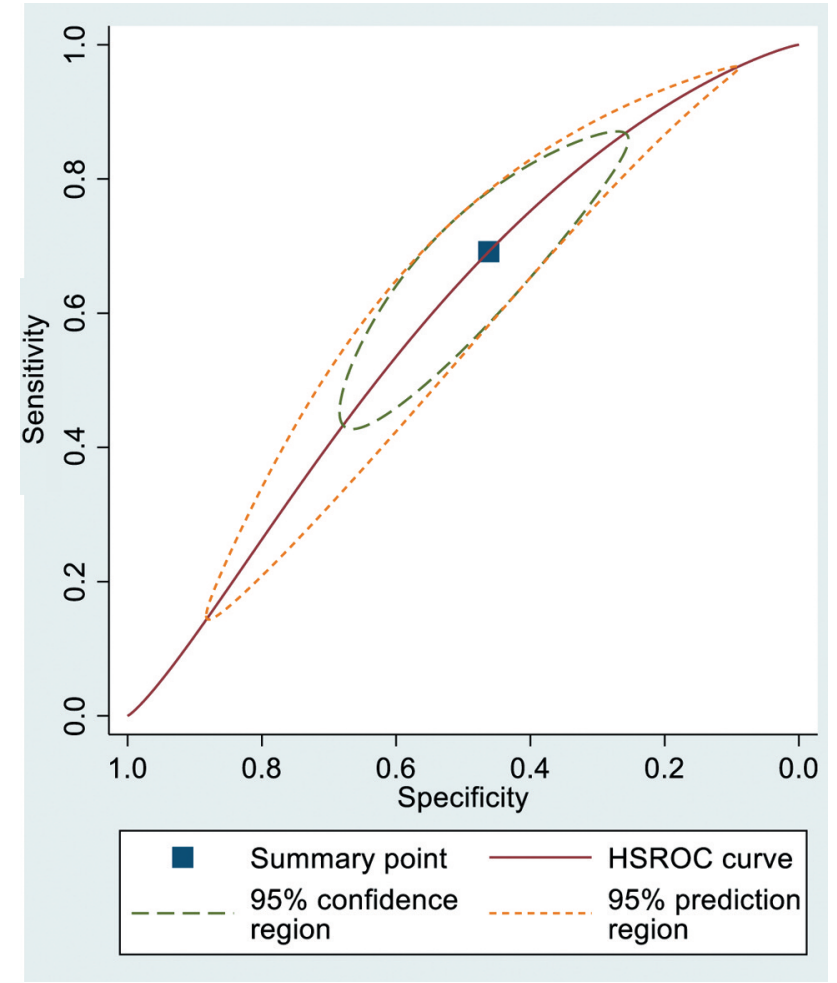

Figure 8. Hierarchical summary receiver operating characteristic (HSROC) curve of the automatically analyzed (AA) screening tests for the colorectal adenoma endpoint. with both $\mathrm{Hb}$ and $\mathrm{Hb} / \mathrm{Hp}$ complex, RIDASCREEN has characteristics that are very similar to those of the CV test, except that the former is a quantitative ELISA test and the latter is a quick test. Not unexpectedly, the AUC values for $\mathrm{Hb}$ and $\mathrm{Hb} / \mathrm{Hp}$ reported by Haug et al. (25): 0.68 (95\% CI=0.65$0.71)$ and $0.64(95 \% \mathrm{CI}=0.61-0.67)$, respectively, are only slightly higher than those obtained in the present study with the AA reading mode of the $\mathrm{CV}$ (Figure 8).

HSROC analysis has become a convenient approach to evaluate the diagnostic accuracy of various diagnostic tests with different components that can be treated like study IDs in the HSROC analysis (27-31). Figure 7 shows the HSROC curve for the VA reading mode in CRA diagnosis, with a reasonably modest AUC value of 0.551 (95\% CI $=0.500-0.602)$. The diagnostic accuracy of the AA reading mode is significantly higher than that of the VA mode, with AUC $=0.606(95 \% \mathrm{CI}=0.550-0.662)(p=0.0160)$ (Figure 8). This makes the AA reading mode a preferred method of interpreting the results of the $\mathrm{CV}$ test, particularly with the CRA endpoint, where the diagnostic accuracy of the $\mathrm{CV}$ test is markedly inferior as compared with the CRC endpoint (32).

\section{Conclusion}

The CV quick test interpreted using the automatic reading mode (AA) test showed significantly higher diagnostic accuracy for the CRA endpoint than did the VA reading mode, or the obsolete guaiac-based HemoccultSENSA test. As pointed out, caution should be followed while comparing the results of different quantitative FIT tests, because different commercial products have different analytical characteristics, and direct comparisons might be misleading. The limitation of this study is the colonoscopy-referral setting, because these patients represent a population with markedly higher CRA prevalence as compared with a native screening setting of asymptomatic subjects. These results clearly implicate that CRA screening by the $\mathrm{CV}$ quick test in its AA mode has the major benefit of avoiding unnecessary endoscopy or radiological procedures while keeping the false positive results at minimum.

\section{Conflicts of Interest}

Tapani Tiusanen, $\mathrm{PhD}$, is an employee of Biohit Company, Helsinki, Finland. The other Authors report no conflicts of interest or financial ties in relation to this study. 


\section{Authors' Contributions}

All Authors contributed to the collection and analysis of data, drafting and revising the manuscript, and read and approved the final article.

\section{Acknowledgements}

The study was funded by the Päivikki and Sakari Sohlberg Foundation, Helsinki, Finland.

\section{References}

1 Mandel JS, Bond JH, Church TR, Snover DC, Bradley GM, Schuman LM and Ederer F: Reducing mortality from colorectal cancer by screening for fecal occult blood. Minnesota Colon Cancer Control Study. N Engl J Med 328(19): 1365-1371, 1993. PMID: 8474513. DOI: 10.1056/NEJM199305133281901

2 Kewenter J, Brevinge H, Engarås B, Haglind E and Ahrén C: Results of screening, rescreening, and follow-up in a prospective randomized study for detection of colorectal cancer by fecal occult blood testing. Results for 68,308 subjects. Scand J Gastroenterol 29(5): 468-473, 1994. PMID: 8036464. DOI: $10.3109 / 00365529409096840$

3 Kronborg O, Fenger C, Olsen J, Jørgensen OD and Søndergaard $\mathrm{O}$ : Randomised study of screening for colorectal cancer with faecal-occult-blood test. Lancet 348(9040): 1467-1471, 1996. PMID: 8942774. DOI: 10.1016/S0140-6736(96)03430-7

4 Hardcastle JD, Chamberlain JO, Robinson MH, Moss SM, Amar SS, Balfour TW, James PD and Mangham CM: Randomised controlled trial of faecal-occult-blood screening for colorectal cancer. Lancet 348(9040): 1472-1477, 1996. PMID: 8942775. DOI: 10.1016/S0140-6736(96)03386-7

5 Faivre J, Dancourt V, Lejeune C, Tazi MA, Lamour J, Gerard D, Dassonville F and Bonithon-Kopp C: Reduction in colorectal cancer mortality by fecal occult blood screening in a French controlled study. Gastroenterology 126(7): 1674-1680, 2004. PMID: 15188160. DOI: 10.1053/j.gastro.2004.02.018

6 Lindholm E, Brevinge $\mathrm{H}$ and Haglind E: Survival benefit in a randomized clinical trial of faecal occult blood screening for colorectal cancer. Br J Surg 95(8): 1029-1036, 2008. PMID: 18563785. DOI: $10.1002 /$ bjs.6136

7 Halloran S, Launoy G and Zappa M: Faecal Occult Blood Testing. In: European Guidelines for Quality Assurance in Colorectal Cancer Screening and Diagnosis. Segnan N, Patrick $\mathrm{J}$ and von Karsa L (eds.). Luxemburg, Publications Office of the European Union, pp. 103-144, 2010.

8 Allison JE, Tekawa IS, Ransom LJ and Adrain AL: A comparison of fecal occult-blood tests for colorectal-cancer screening. N Engl J Med 334(3): 155-159, 1996. PMID: 8531970. DOI: 10.1056/NEJM199601183340304

9 Zappa M, Castiglione G, Paci E, Grazzini G, Rubeca T, Turco $\mathrm{P}$, Crocetti $\mathrm{E}$ and Ciatto S: Measuring interval cancers in population-based screening using different assays of fecal occult blood testing: the District of Florence experience. Int J Cancer 92(1): 151-154, 2001. PMID: 11279619.

10 Hol L, Wilschut JA, van Ballegooijen M, van Vuuren AJ, van der Valk H, Reijerink JC, van der Togt AC, Kuipers EJ, Habbema JD and van Leerdam ME: Screening for colorectal cancer: random comparison of guaiac and immunochemical faecal occult blood testing at different cut-off levels. Br J Cancer 100(7): 1103-1110, 2009. PMID: 19337257. DOI: 10.1038/sj.bjc.6604961

11 Vasilyev S, Smirnova E, Popov D, Semenov A, Eklund C, Hendolin P, Paloheimo L and Syrjänen K: A new-generation fecal immunochemical test (FIT) is superior to quaiac-based test in detecting colorectal neoplasia among colonoscopy referral patients. Anticancer Res 35(5): 2873-2880, 2015. PMID: 25964570.

12 Guimarães DP, Fregnani JH, Reis RM, Taveira LN, Scapulatempo-Neto C, Matsushita M, Silva SRM, Oliveira CZ, Longatto-Filho A, Eklund C, Paloheimo L, Mauad E, Suovaniemi O and Syrjänen K: Comparison of a new-generation fecal immunochemical test (FIT) with guaiac fecal occult blood test (gFOBT) in detecting colorectal neoplasia among colonoscopy-referral patients. Anticancer Res 39(1): 261-269, 2019. PMID: 30591467. DOI: 10.21873/anticanres.13106

13 Federici A, Giorgi Rossi P, Borgia P, Bartolozzi F, Farchi S and Gausticchi G: The immunochemical faecal occult blood test leads to higher compliance than the guaiac for colorectal cancer screening programmes: a cluster randomized controlled trial. J Med Screen 12(2): 83-88, 2005. PMID: 15949119. DOI: 10.1258/0969141053908357

14 Suovaniemi O: Immunoassay for fecal human hemoglobin. Available at: https://patents.google.com/patent/US4427769 [Last accessed on October 13, 2021]

15 Meklin J, Syrjänen K and Eskelinen M: Colorectal cancer screening with traditional and new-generation fecal immunochemical tests: a critical review of fecal occult blood tests. Anticancer Res 40(2): 575-581, 2020. PMID: 32014898. DOI: 10.21873 /anticanres.13987

16 Meklin J, Syrjänen K and Eskelinen M: Fecal occult blood tests in colorectal cancer screening: Systematic review and metaanalysis of traditional and new-generation fecal immunochemical tests. Anticancer Res 40(7): 3591-3604, 2020. PMID: 32620599. DOI: 10.21873/anticanres.14349

17 Songster CL, Barrows GH and Jarrett DD: Immunochemical detection of fecal occult blood-the fecal smear punch-disc test: a new non-invasive screening test for colorectal cancer. Cancer $45(5$ Suppl): 1099-1102, 1980. PMID: 6965607. DOI: 10.1002/10970142(19800315)45:5+<1099::aid-cncr2820451312>3.0.co;2-t

18 BioHit HealthCare. ColonView Quick Test. Available at: https://www.biohithealthcare.com/en/products/diagnostictests/colonview-quick-test/ [Last accessed on October 13, 2021]

19 Meklin J, Eskelinen M, Syrjänen K and Eskelinen M: Leucocyte count does not improve the diagnostic performance of a diagnostic score (DS) in distinguishing acute appendicitis (AA) from nonspecific abdominal pain (NSAP). In Vivo 34(6): $3327-$ 3339, 2020. PMID: 33144440. DOI: 10.21873/invivo.12171

20 Meklin J, Eskelinen M, Syrjänen K and Eskelinen M: Genderspecific performance of a diagnostic score in acute appendicitis. In Vivo 34(6): 3687-3703, 2020. PMID: 33144486. DOI: 10.21873/invivo.12217

21 Castiglione G, Zappa M, Grazzini G, Sani C, Mazzotta A, Mantellini $\mathrm{P}$ and Ciatto $\mathrm{S}$ : Cost analysis in a population based screening programme for colorectal cancer: comparison of immunochemical and guaiac faecal occult blood testing. J Med Screen 4(3): 142-146, 1997. PMID: 9368871. DOI: 10.1177/09691 4139700400306

22 Fenton JJ, Elmore JG, Buist DS, Reid RJ, Tancredi DJ and Baldwin LM: Longitudinal adherence with fecal occult blood 
test screening in community practice. Ann Fam Med 8(5): $397-$ 401, 2010. PMID: 20843880. DOI: 10.1370/afm.1133

23 Allison JE, Sakoda LC, Levin TR, Tucker JP, Tekawa IS, Cuff T, Pauly MP, Shlager L, Palitz AM, Zhao WK, Schwartz JS, Ransohoff DF and Selby JV: Screening for colorectal neoplasms with new fecal occult blood tests: update on performance characteristics. J Natl Cancer Inst 99(19): 1462-1470, 2007. PMID: 17895475 . DOI: $10.1093 /$ jnci/djm150

24 Hundt S, Haug U and Brenner H: Comparative evaluation of immunochemical fecal occult blood tests for colorectal adenoma detection. Ann Intern Med 150(3): 162-169, 2009. PMID: 19189905. DOI: 10.7326/0003-4819-150-3-200902030-00005

25 Haug U, Hundt S and Brenner H: Quantitative immunochemical fecal occult blood testing for colorectal adenoma detection: evaluation in the target population of screening and comparison with qualitative tests. Am J Gastroenterol 105(3): 682-690, 2010. PMID: 19953091. DOI: 10.1038/ajg.2009.668

26 Lee JK, Liles EG, Bent S, Levin TR and Corley DA: Accuracy of fecal immunochemical tests for colorectal cancer: systematic review and meta-analysis. Ann Intern Med 160(3): 171, 2014. PMID: 24658694. DOI: 10.7326/M13-1484

27 Eskelinen M, Meklin J, Syrjänen $\mathrm{K}$ and Eskelinen M: Performance of a diagnostic score in confirming acute cholecystitis among patients with acute abdominal pain. Anticancer Res 40(12): 6947-6956, 2020. PMID: 33288589. DOI: 10.21873 /anticanres.14719
28 Eskelinen M, Meklin J, Syrjänen K and Eskelinen M: Pediatric acute appendicitis score in children with acute abdominal pain (AAP). Anticancer Res 41(1): 297-306, 2021. PMID: 33419824. DOI: 10.21873 /anticanres.14776

29 Eskelinen M, Meklin J, Syrjänen K and Eskelinen M: A diagnostic score (DS) is a powerful tool in diagnosis of acute appendicitis in elderly patients with acute abdominal pain. Anticancer Res 41(3): 1459-1469, 2021. PMID: 33788738. DOI: 10.21873/anticanres.14904

30 Eskelinen M, Meklin J, Syrjänen K and Eskelinen M: A diagnostic score for acute small bowel obstruction. Anticancer Res 41(4): 19591970, 2021. PMID: 33813402. DOI: 10.21873/anticanres.14963

31 Eskelinen M, Meklin J, Syrjänen K and Eskelinen M: A diagnostic score for reliable confirmation of acute renal colic among patients with acute abdominal pain. Anticancer Res 41(6): 3045-3054, 2021. PMID: 34083296. DOI: 10.21873/anticanres.15087

32 Meklin J, Eskelinen M, Guimaraes DP, Selander T, Inkinen J, Tiusanen T, Syrjänen $\mathrm{K}$ and Eskelinen $\mathrm{M}$ : The new generation immunochemical test for fecal occult blood (ColonView Quick Test) shows a high diagnostic accuracy in colorectal cancer detection. Anticancer Res 41(10): 5071-5079, 2021. PMID: 34593457. DOI: 10.21873 /anticanres. 15323

Received September 24, 2021

Revised October 13, 2021

Accepted October 14, 2021 